\title{
On the growth of quotients of Kleinian groups
}

\author{
FRANÇOISE DAL'BO $\dagger$, MARC PEIGNÉ $\ddagger$ JEAN-CLAUDE PICAUD $\ddagger$ and \\ ANDREA SAMBUSETTI§ \\ $\dagger$ IRMAR, Université de Rennes-I, Campus de Beaulieu, \\ 35042 Rennes Cedex, France \\ (e-mail: francoise.dalbo@univ-rennes1.fr) \\ † LMPT, UMR 6083, Faculté des Sciences et Techniques, Parc de Grandmont, \\ 37200 Tours, France \\ (e-mail: peigne@univ-tours.fr, jean-claude.picaud@univ-tours.fr) \\ $\S$ Istituto di Matematica G. Castelnuovo Sapienza Università di Roma, P.le Aldo Moro 5, \\ 00185 Roma, Italy \\ (e-mail: sambuset@mat.uniromal.it)
}

(Received 24 June 2009 and accepted in revised form 27 January 2010)

\begin{abstract}
We study the growth and divergence of quotients of Kleinian groups $G$ (i.e. discrete, torsionless groups of isometries of a Cartan-Hadamard manifold with pinched negative curvature). Namely, we give general criteria ensuring the divergence of a quotient group $\bar{G}$ of $G$ and the 'critical gap property' $\delta_{\bar{G}}<\delta_{G}$. As a corollary, we prove that every geometrically finite Kleinian group satisfying the parabolic gap condition (i.e. $\delta_{P}<\delta_{G}$ for every parabolic subgroup $P$ of $G$ ) is growth tight. These quotient groups naturally act on non-simply connected quotients of a Cartan-Hadamard manifold, so the classical arguments of Patterson-Sullivan theory are not available here; this forces us to adopt a more elementary approach, yielding as by-product a new elementary proof of the classical results of divergence for geometrically finite groups in the simply connected case. We construct some examples of quotients of Kleinian groups and discuss the optimality of our results.
\end{abstract}

\section{Introduction}

A Kleinian group $G$ is a discrete, non-elementary, torsion-free group of isometries of an $n$-dimensional Cartan-Hadamard space $X$ with pinched negative curvature (i.e. a complete, simply connected Riemannian manifold with sectional curvature bounded between two negative constants $-K_{0}^{2} \leq k_{X} \leq-k_{0}^{2}$ ). In this paper, we shall be concerned with quotients of such groups. Considering quotients of $G$ is equivalent to considering all possible normal coverings of the manifold $X_{0}=G \backslash X$; actually, any quotient group $\bar{G}=N \backslash G$ by a normal subgroup is the automorphism group of the Riemannian 
covering $\bar{X}=N \backslash X \rightarrow X_{0}$, endowed with the quotient distance

$$
\bar{d}(\overline{\mathbf{x}}, \overline{\mathbf{y}})=\inf \{d(\mathbf{x}, n \mathbf{y}) ; n \in N\}
$$

for $\mathbf{x} \in \overline{\mathbf{x}}, \mathbf{y} \in \overline{\mathbf{y}}$, where $d$ is the Riemannian distance of $X$.

The normal coverings of $X_{0}$ form a countable family $\operatorname{Cov}\left(X_{0}\right)$. Then, every fixed Riemannian invariant gives rise, when applied to elements of $\operatorname{Cov}\left(X_{0}\right)$, to a whole spectrum of associated numbers: for instance, the spectrum $\operatorname{Ent}\left(X_{0}\right)$ of volume entropies of all normal coverings of $X_{0}$, or the spectrum $\operatorname{Exp}\left(X_{0}\right)$ of critical exponents of their automorphism groups.

Recall that the volume entropy of a covering $\bar{X}$ and the critical exponent of its automorphism group $\bar{G}$ are the asymptotic invariants defined respectively as

$$
\begin{gathered}
\omega(\bar{X})=\limsup _{R \rightarrow+\infty} \frac{1}{R} \ln \operatorname{vol}\left(\mathcal{B}_{\bar{X}}(\overline{\mathbf{x}}, R)\right), \\
\delta_{\bar{G}}=\limsup _{R \rightarrow+\infty} \frac{1}{R} \ln \sharp\left\{\left.\bar{g} \in \overline{,}^{-} G\right|^{-} d(\overline{\mathbf{x}}, \bar{g} \overline{\mathbf{x}}) \leq R\right\}
\end{gathered}
$$

where $\mathcal{B}_{\bar{X}}(\overline{\mathbf{x}}, R)$ denotes the ball of radius $R$ in $\bar{X}$ centred at $\overline{\mathbf{x}}$; see $[\mathbf{2}, \mathbf{7}, \mathbf{1 1}, \mathbf{1 5}]$. Both numbers are independent of the choice of the point $\overline{\mathbf{x}} \in \bar{X}$; moreover, the critical exponent $\delta_{\bar{G}}$ coincides with the abscissa of convergence of the Poincaré series of $\bar{G}$,

$$
\mathcal{P}_{\bar{G}}(s, \overline{\mathbf{x}})=\sum_{\bar{g} \in \bar{G}} e^{-s \bar{d}(\overline{\mathbf{x}}, \bar{g} \overline{\mathbf{x}})}, \quad s \in \mathbb{R}^{+},
$$

and, if $k_{X} \geq-K_{0}^{2}$, we know that

$$
\delta_{\bar{G}} \leq \omega(\bar{X}) \leq(n-1) K_{0}<+\infty
$$

by the standard comparison theorems of Riemannian geometry.

The invariants $\omega(\bar{X})$ and $\delta_{\bar{G}}$ (hence, the aforementioned spectra $\operatorname{Ent}\left(X_{0}\right)$ and $\operatorname{Exp}\left(X_{0}\right)$ ) coincide if $X_{0}$ is compact, but they may differ when $X_{0}$ has finite volume (see [7]). The critical exponent is considerably more interesting than volume entropy when $G$ has infinite volume: for instance, for every geometrically finite hyperbolic manifold $X_{0}=G \backslash \mathbb{H}$ of infinite volume, the spectrum of entropies $\operatorname{Ent}\left(X_{0}\right)$ is always reduced to the value $n-1$ (since $X_{0}$ and all its coverings contain a funnel); on the other hand, the spectrum of critical exponents $\operatorname{Exp}\left(X_{0}\right)$ is a particular subset of the interval [0,n-1] which strongly depends on $X_{0}$, and whose top value $\delta_{G}$ is related to the dynamics of the geodesic flow on $U X_{0}$.

In this paper we shall be interested in the spectrum $\operatorname{Exp}\left(X_{0}\right)$, namely in comparing $\delta_{\bar{G}}$ with $\delta_{G}$. We shall say that $\bar{G}$ satisfies the critical gap property when the strict inequality $\delta_{\bar{G}}<\delta_{G}$ holds, and that $G$ is growth tight when $\delta_{\bar{G}}<\delta_{G}$ for any proper quotient $\bar{G}$ of $G$; that is, when the top value $\delta_{G}$ of the spectrum of critical exponents $\operatorname{Exp}\left(X_{0}\right)$ is a value of multiplicity one.

The notion of growth tightness was introduced by Grigorchuk and de la Harpe in [10] for finitely generated groups endowed with word metrics; in this context, the fourth author [17] proved growth tightness for amalgamated free products (exhibiting the first examples of groups of exponential growth whose minimal growth is not attained by any 
generating set), while Arzhantseva and Lysenok in [1] proved the same property for Gromov hyperbolic groups without finite normal subgroups. Growth tightness was first investigated in the Riemannian setting in [16], for hyperbolic surfaces, and in [18] for general cocompact Kleinian groups, where the fourth author shows that a positive critical gap $\Delta(G, \bar{G}):=\delta_{G}-\delta_{\bar{G}}>0$ produces estimates on the systole and on the bottom of the spectrum of the covering $\bar{X}$.

Let us stress the fact that, though growth tightness of groups with respect to word metrics and with respect to Riemannian metrics are closely related problems, neither of them can be deduced from the other (not even for cocompact groups) as the critical exponent is not invariant by quasi-isometries.

Our study is on the stream of [18], and we consider here the case of geometrically finite groups: these are the Kleinian groups $G$ acting on some $\epsilon$-neighbourhood of the convex hull of their limit set $\Lambda_{G}$ with quotient of finite volume (see $\S 3$ for details and their main properties). As we will see, growth tightness no longer holds in general due to the presence of parabolic elements, but it is still true for most of them (see Theorem 1.4 below).

We will see that, for a proper quotient $\bar{G}$ of $G$, the positivity of the critical gap $\Delta(G, \bar{G})$ is related to the divergence of $\bar{G}$, which means that $\mathcal{P}_{\bar{G}}\left(\delta_{\bar{G}}, \overline{\mathbf{x}}\right)=+\infty$ (otherwise $\bar{G}$ is said to be convergent). This sheds new light on the divergence property of a group. It is now classic that the divergence/convergence of $G$ is of interest when studying the ergodic properties of the geodesic flow $\left(\phi_{t}\right)_{t}$ on the unit tangent bundle of the quotient manifold $G \backslash X$; namely, the divergence of $G$ is a necessary condition for the existence and unicity of an invariant probability measure of maximal entropy for $\left(\phi_{t}\right)_{t}$ [12]. It is also of interest when comparing the critical exponent of a Kleinian group $G$ with that of a subgroup $H$ : if $H$ is divergent and its limit set $\Lambda_{H}$ is a proper subset of $\Lambda_{G}$, then the strict inequality $\delta_{G}>\delta_{H}$ holds [6].

In general, it is not easy to decide wether or not a Kleinian group $G$ acting on $X$ is convergent or divergent; quite surprisingly, there exist only partial answers to this natural question. It is well known that any convex cocompact group $G$ (i.e. a group acting cocompactly on the convex hull of its limit set $\Lambda_{G}$ ) is divergent [19]. The same problem was studied in the case of geometrically finite groups; these groups are not so far from the convex cocompact groups, nevertheless the tools to investigate them are more sophisticated. Sullivan proved that any geometrically finite group $G$ of constant curvature is divergent, using refined arguments based on Patterson-Sullivan measures [19]. The same approach in [6] allowed Dal'Bo et al to extend this result to the variable curvature case, under mild hypotheses on the maximal parabolic subgroups: a geometrically finite group $G$ is divergent if for every parabolic subgroup $P$ one has $\delta_{P}<\delta_{G}$. In the present text we obtain, as a by-product, an elementary proof of this result (Theorem 1.2), which does not require the theory of Patterson-Sullivan measures.

Actually, Patterson-Sullivan's theory gives an analytical description of the way the orbit $G \mathbf{x}$ accumulates on the limit set, via Sullivan's shadowing lemma. Now this lemma is related to the fact that visual and Busemann compactifications of $X$ coincide, so that the action of $G$ on $X$ can be 'read' through the action on the boundary; but this identification is no longer true when considering a non-simply connected manifold $\bar{X}=N \backslash X$ (for 
instance, there may exist fellow geodesics going to infinity but yielding different Busemann functions), so that the notion of shadow which is central in Patterson-Sullivan is not relevant here.

For a given quotient $\bar{G}=N \backslash G$ of $G$ and any subgroup $H$ of $G$, we shall denote by $\bar{H}=(H \cap N) \backslash H$ the quotient subgroup. Then, we shall say that $G(\bar{G})$ satisfies the parabolic gap condition, denoted by $(P G C)$, if $\delta_{P}<\delta_{G}\left(\delta_{\bar{P}}<\delta_{\bar{G}}\right)$ for all parabolic subgroups $P$ of $G$. We are then in position to state the main results of this paper.

THEOREM 1.1. (Critical gap criterion) Let $G$ be a Kleinian group of a Cartan-Hadamard manifold with pinched negative curvature, and let $\bar{G}=N \backslash G$ be a proper quotient of $G$. If $\bar{G}$ is divergent, then $\delta_{\bar{G}}<\delta_{G}$.

As will be clear from the proof, the gap $\delta_{A *\{h\}}-\delta_{A}$ will depend on the length of the shortest closed geodesic of $\bar{X}$, but we do not concentrate on this point in this work.

Theorem 1.1 suggests that growth tightness and divergence of quotient groups are related problems; in this perspective, we prove the following criterion of divergence for quotients of geometrically finite groups generalizing the aforementioned Dal'Bo et al result $[6]$.

THEOREM 1.2. (Divergence criterion) Let $G$ be a geometrically finite Kleinian group of a Cartan-Hadamard manifold with pinched negative curvature, and let $\bar{G}=N \backslash G$ be a quotient of $G$. If $\bar{G}$ satisfies the parabolic gap condition, then $\bar{G}$ is divergent.

Remark 1.3. We will see in Example 1 of $\S 5$ that, when $(P G C)$ fails for $\bar{G}$, then the group $\bar{G}$ may well be of convergent type. Example 1 will also show that when $\bar{G}$ is convergent then the equality $\delta_{\bar{G}}=\delta_{G}$ may occur.

It may be of interest to stress the generality of the above theorem; actually, $\bar{G}$ can be virtually any finitely generated group, since any finitely generated group can be represented as the quotient of a free Schottky (hence geometrically finite) group of $\mathbb{H}^{2}$.

Combining Theorems 1.1 and 1.2, we deduce the following theorem.

THEOREM 1.4. (Growth tightness of geometrically finite groups) Let $G$ be $a$ geometrically finite Kleinian group of a Cartan-Hadamard manifold with pinched negative curvature. If $G$ is convex cocompact or $G$ satisfies the parabolic gap condition, then $G$ is growth tight, i.e. $\delta_{\bar{G}}<\delta_{G}$ for any proper quotient $\bar{G}$ of $G$.

In other words, the universal covering of a geometrically finite manifold $X_{0}=G \backslash X$, with $G$ satisfying $(P G C)$, is characterized by the property of being the normal covering whose automorphism group has maximal critical exponent.

An interesting question which remains unsettled is whether the top value $\delta_{G}$ of the spectrum $\operatorname{Exp}\left(X_{0}\right)$ of critical exponents of a geometrically finite manifold is isolated, that is,

$$
\Delta(G):=\inf _{\bar{G} \neq G}\left\{\delta_{G}-\delta_{\bar{G}}\right\}>0,
$$

or possibly to find examples where $\Delta(G)=0$. 
Remarks 1.5.

(i) As will be clear from the Examples 2-3 of $\$ 5$, the divergence and condition $(P G C)$ for $G$ are independent of the corresponding properties for $\bar{G}$ : that is, $(P G C$ ) (or divergence) holding for $G$ does not imply that $(P G C)$ (or divergence) is satisfied for $\bar{G}$, and vice versa.

(ii) Recall that the parabolic groups of rank-one symmetric spaces are divergent [5], and that (as a consequence of [6]) this implies condition (PGC); it then follows that all geometrically finite groups of rank-one symmetric spaces are growth tight.

The paper is organized as follows. Section 2 deals with a criterion of comparison for the critical exponent of free products of sets and Schottky groups. Section 3 is devoted to the proof of the critical gap criterion (Theorem 1.1), for a divergent quotient, using a variation of Klein's table-tennis argument. In $\S 4$ we give the proof of the divergence criterion (Theorem 1.2) for geometrically finite groups, showing a quasisubmultiplicativity property for the growth of annuli in the orbits of these groups (see Proposition 4.1); then we use the divergence criterion to prove Theorem 1.4. Finally, in $\S 5$ we give examples of geometrically finite groups $G$ with quotients $\bar{G}$ in the following, distinct situations:

(1) $\bar{G}$ is convergent and $\delta_{\bar{G}}=\delta_{G}$;

(2) (PGC) holds for $G$ (divergent), but not for $\bar{G}$ (convergent);

(3) conversely, $(P G C)$ holds for $\bar{G}$ (divergent), but not for $G$ (convergent).

\section{Critical exponent comparison for free products}

In this section, we establish a simple criterion of critical gap for free products of sets of isometries $A * B$ acting on $X$, which we will use later. We will need the following, classical facts on the geometry of a Cartan-Hadamard manifold $X$ of strictly negative curvature $k_{X} \leq-k_{0}^{2}$ (see, for instance, [9]). In the following, we shall always use the notation [x, y] for a geodesic segment joining $\mathbf{x}, \mathbf{y}$.

FACT 2.1. For any $\theta>0$, there exists a constant $c=c\left(\theta, k_{0}\right)>0$ such that every geodesic triangle $T$ in $X$ with vertices $\mathbf{x}, \mathbf{y}, \mathbf{z} \in X$ and angle at $\mathbf{y}$ greater than $\theta$ satisfies $d(\mathbf{x}, \mathbf{z}) \geq$ $d(\mathbf{x}, \mathbf{y})+d(\mathbf{y}, \mathbf{z})-c$.

FACT 2.2. There exists a function $\epsilon=\epsilon\left(k_{0}, L\right)$ such that if $\gamma$ is a curve in $X$ with endpoints $\mathbf{x}, \mathbf{y}$ and whose length satisfies $\ell(\gamma) \leq \ell([\mathbf{x}, \mathbf{y}])+L$, then $\gamma$ is included in the closed $\epsilon$-neighbourhood of $[\mathbf{x}, \mathbf{y}]$.

Recall that any $h \in \operatorname{Isom}(X)$ without fixed points in $X$ has one or two fixed points on the boundary at infinity $X(\infty)$ and, accordingly, $h$ is respectively called parabolic or hyperbolic. When $h$ is parabolic, we will denote by $\xi_{h}$ its unique fixed point (i.e. $\xi_{h}:=$ $\lim _{n \rightarrow \pm \infty} h^{n} \cdot \mathbf{x}$ for any $\mathbf{x} \in X$ ), while when $h$ is hyperbolic we will denote its repulsive and attracting fixed points respectively by $\xi_{h}^{-}, \xi_{h}^{+}$(i.e. $\xi_{h}^{ \pm}:=\lim _{n \rightarrow \pm+\infty} h^{ \pm n} \cdot \mathbf{x}$, for any $\mathbf{x} \in X)$. The axis of a hyperbolic element $h$ will be denoted by $\tilde{h}=\left[\xi_{h}^{-}, \xi_{h}^{+}\right]$, and we let $|h|=d(x, h x), x \in \tilde{h}$, the displacement of $h$ along its axis. 
FACT 2.3. Let $h$ be a hyperbolic isometry of $X$, and let $V^{-}$and $V^{+}$be two neighbourhoods of $\xi_{h}^{-}$and $\xi_{h}^{+}$respectively in $X(\infty)$. Then, there exists $n_{0} \geq 1$ such that $h^{n}\left(X(\infty) \backslash V^{-}\right) \subset V^{+}$and $h^{-n}\left(X(\infty) \backslash V^{+}\right) \subset V^{-}$for any $n \geq n_{0}$.

Now let $A$ and $B$ be two non-empty subsets of $\operatorname{Isom}(X)$ intersecting at most in the identity. We set $A^{*}:=A \backslash\{\mathrm{Id}\}, B^{*}:=B \backslash\{\mathrm{Id}\}$, and we say that a finite sequence (a word) $\left(g_{1}, \ldots, g_{n}\right)$ on the alphabet $A^{*} \cup B^{*}$ is admissible with respect to $(A, B)$ if, for all $i, g_{i}$ and $g_{i+1}$ do not belong to the same set $A$ or $B$. We will denote by $\mathfrak{A}(A, B)$ the union of such admissible sequences, for any $n \in \mathbb{N}^{*}$, and by $A * B$ the subset of $\operatorname{Isom}(X)$ defined by

$$
A * B:=\left\{g_{1} \cdots g_{n} \mid\left(g_{i}\right)_{1 \leq i \leq n} \in \mathfrak{A}(A, B), n \geq 1\right\} \cup\{\mathrm{Id}\} .
$$

The critical exponent makes sense for any general countable subset $S$ of $\operatorname{Isom}(X)$ (and not only for discrete subgroups), and is the abscissa of convergence $\delta_{S}$ of the associated 'Poincaré series' $\mathcal{P}_{S}(s, \mathbf{x})=\sum_{g \in S} \exp (-s d(\mathbf{x}, g \cdot \mathbf{x}))$, where $\mathbf{x} \in X$ and $s \in \mathbb{R}$. With this notation, we clearly have $\delta_{A * B} \geq \max \left(\delta_{A}, \delta_{B}\right)$; the question whether or not the inequality is strict is more delicate.

CRITERION 2.4. Let $A$ be a countable subset of $\operatorname{Isom}(X)$ and $h$ a non-trivial isometry of $X, h \notin A$. Assume that the natural evaluation map

$$
\begin{aligned}
\phi: \mathfrak{A}(A,\{h\}) & \rightarrow A *\{h\} \\
\left(g_{i}\right)_{1 \leq i \leq n} & \mapsto g_{1} \cdots g_{n}
\end{aligned}
$$

is one-to-one. If $\mathcal{P}_{A}(s, \mathbf{x})$ diverges at its critical exponent, then $\delta_{A *\{h\}}>\delta_{A}$.

Proof. Since $\phi$ is one-to-one, for any $s \geq 0$ and $\mathbf{x} \in X$ we get

$$
\begin{aligned}
\sum_{g \in A *\{h\}} \exp (-s d(\mathbf{x}, g \mathbf{x})) & \geq \sum_{k \geq 1} \sum_{a_{1}, \ldots, a_{k} \in A^{*}} \exp \left(-s d\left(\mathbf{x}, a_{1} h a_{2} h \cdots a_{k} h \mathbf{x}\right)\right) \\
& \geq \sum_{k \geq 1} \sum_{a_{1}, \ldots, a_{k} \in A^{*}} \exp \left(-s\left[d\left(\mathbf{x}, a_{1} h \mathbf{x}\right)+\cdots+d\left(\mathbf{x}, a_{k} h \mathbf{x}\right)\right]\right) \\
& \geq \sum_{k \geq 1}\left(\sum_{a \in A^{*}} \exp (-s d(\mathbf{x}, a \mathbf{y}))\right)^{k}
\end{aligned}
$$

for $\mathbf{y}=h \mathbf{x}$. As $\sum_{a \in A^{*}} \exp \left(-\delta_{A} d(\mathbf{x}, a \mathbf{x})\right)=+\infty$ at $s=\delta_{A}$, we may choose $s_{0}>\delta_{A}$ such that $\sum_{a \in A^{*}} \exp \left(-s_{0} d(\mathbf{x}, a \mathbf{y})\right)>1$, so $\sum_{g \in A *\{h\}} \exp (-s d(\mathbf{x}, g \cdot \mathbf{x}))$ diverges for $s=s_{0}$. It then follows that $\delta_{A *\{h\}} \geq s_{0}>\delta_{A}$.

\section{The critical gap property for divergent quotients}

Let $G$ be a Kleinian group of a Cartan-Hadamard manifold $X$ of pinched, negative curvature, and let $\bar{G}=N \backslash G$ be the quotient of $G$ by a normal subgroup $N$. In the following we shall systematically use the following notation:

- $\quad \bar{X}$ for the quotient manifold $N \backslash X$, with Riemannian distance $\bar{d}$ given by the quotient distance of $d$;

- $\pi: X \rightarrow \bar{X}, \rho: G \rightarrow \bar{G}$ for the natural projections, but we shall often use $\overline{\mathbf{x}}=\pi(\mathbf{x})$ and $\bar{g}=\rho(g)=g N$ for the projections of $\mathbf{x} \in X, g \in G$ on $\bar{X}, \bar{G}$ respectively; 
- $G_{N}$ for a symmetric set of minimal representatives of $\bar{G}$ in $G$, which means that for any $\bar{g} \in \bar{G}$, there exists a unique $g \in G_{N}$ such that $\pi(g)=\bar{g}$ and

$$
g^{-1} \in G_{N} \quad d(\mathbf{o}, g \mathbf{o})=\bar{d}(\overline{\mathbf{o}}, \bar{g} \overline{\mathbf{o}})
$$

(notice that $\mathrm{Id} \in G_{N}$, and the first condition can be satisfied since $N$ is normal).

We need to establish a ping-pong lemma for a non-trivial quotient of a Kleinian group.

LeMma 3.1. Let $h \in N$ be a hyperbolic isometry and let $\mathbf{o}$ belong to its axis $\tilde{h}$. There exist two disjoint compact neighbourhoods $W^{-}$and $W^{+}$in $X \cup X(\infty)$ of $\xi_{h}^{-}$and $\xi_{h}^{+}$ respectively, and numbers $p_{0} \in \mathbb{N}^{*}, \theta_{0} \in \mathbb{R}^{+*}$ such that:

(i) $G_{N} \mathbf{0} \cap\left(W^{-} \cup W^{+}\right)=\emptyset$;

(ii) for any $g \in G_{N}$ and $w \in\left(W^{-} \cup W^{+}\right)$, we have $g w \notin\left(W^{-} \cup W^{+}\right)$;

(iii) for any $g \in G_{N}^{*}$ and any $w \in\left(W^{-} \cup W^{+}\right)$, we have $L_{\mathbf{o}}(g \mathbf{0}, w) \geq \theta_{0}$;

(iv) for any $p \geq p_{0}$, we have

$$
h^{p}\left(\left(X \cup X(\infty) \backslash W^{-}\right)\right) \subset W^{+} \quad \text { and } \quad h^{-p}\left(\left(X \cup X(\infty) \backslash W^{-}\right)\right) \subset W^{+} .
$$

Proof. (i) Suppose that there exists a sequence $\left(g_{p}\right)_{p \geq 1}$ in $G_{N}$ such that $\left(g_{p} \mathbf{0}\right)_{p \geq 1}$ converges to $\xi_{h}^{+}$. By minimality, $d\left(\mathbf{o}, g_{p} \mathbf{o}\right) \leq d\left(n \mathbf{o}, g_{p} \mathbf{o}\right)$ for any $n \in N$, and consequently we have

$$
d(\mathbf{o}, h \cdot \mathbf{o})=B_{\xi_{h}^{+}}(\mathbf{o}, h \mathbf{o})=\lim _{p \rightarrow+\infty}\left(d\left(\mathbf{o}, g_{p} \mathbf{o}\right)-d\left(h \mathbf{o}, g_{p} \mathbf{0}\right)\right) \leq 0
$$

where $B_{\xi_{h}^{+}}(\mathbf{o}, \cdot)$ is the Busemann function centred at $\xi_{h}^{+} \in X(\infty)$; this contradicts the fact that $\xi_{h}^{+}$is the attractive fixed point of $h$. The same contradiction appears if we replace $\xi_{h}^{+}$ by $\xi_{h}^{-}$.

(ii) Suppose that (ii) does not hold; we can find sequences $\left(w_{p}\right)_{p \geq 1} \in X \cup X(\infty)$ and $\left(g_{p}\right)_{p \geq 1}$ with $g_{p} \in G_{N}$ such that $w_{p} \rightarrow \xi, g_{p} w_{p} \rightarrow \xi^{\prime}$ with $\xi, \xi^{\prime} \in\left\{\xi_{h}^{+}, \xi_{h}^{-}\right\}$. Moreover, by (i), we know that $g_{p} \mathbf{o} \rightarrow \xi^{\prime \prime} \notin\left\{\xi_{h}^{+}, \xi_{h}^{-}\right\}$. Since $\xi^{\prime} \neq \xi^{\prime \prime}$, the distance between $\mathbf{o}$ and the geodesic $\left[g_{p} \mathbf{o}, g_{p} w_{p}\right]$ (hence $d\left(g_{p}^{-1} \mathbf{o}, \mathbf{o} w_{p}\right)$ ) remains uniformly bounded. But the geodesic passing through $\mathbf{o}$ and $w_{p}$ converges to the axis $\tilde{h}$ so that the limit points of the sequence $\left(g_{p}^{-1} \mathbf{o}\right)_{p \geq 1}$ must necessarily be equal to either $\xi_{h}^{+}$or $\xi_{h}^{-}$. As $G_{N}$ is symmetric, this contradicts (i).

(iii) and (iv). These are respectively consequences of (i) and of Fact 2.3.

Let us now explain the ideas behind the proof of Theorem 1.1. Given the group $G$ and a proper quotient $\bar{G}=N \backslash G$, we choose a hyperbolic isometry $h \in N$ (notice that, $G$ being non-elementary, $N$ always contains a hyperbolic element), a point $\mathbf{o}$ on its axis and a symmetric set of minimal representatives $G_{N}$ as in the above lemma. Then, roughly speaking, by combining alternatively elements of $G_{N}$ with a large power of $h$, we may construct a free subset $G_{N} *\left\{h^{p}\right\}$ of $G$, for $p \gg 0$, whose elements may be written uniquely as admissible words with respect to $\left(G_{N}, h^{p}\right)$. We will then deduce, by Criterion 2.4 , that $\delta_{G} \geq \delta_{G_{N} *\left\{h^{p}\right\}}>\delta_{G_{N}}=\delta_{\bar{G}}$. In order to have the unicity of expression mentioned above, we actually need to take a subset of $G_{N}$ whose elements stay at a large enough distance from each other. We summarize these ideas in the following proposition. 
PROPOSITION 3.2. For any $p \gg 0$, there exists $\lambda=\lambda\left(\theta_{0}, k_{0},|h|, p\right)>0$ such that for any $l, l^{\prime} \geq 1$ and $g_{1}, \ldots, g_{l}, g_{1}^{\prime}, \ldots, g_{l^{\prime}}^{\prime}$ in $G_{N}$ satisfying

$$
g_{1} h^{p} \ldots h^{p} g_{l}=g_{1}^{\prime} h^{p} \ldots h^{p} g_{l^{\prime}}^{\prime}
$$

we have $d\left(g_{1} \mathbf{o}, g_{1}^{\prime} \mathbf{o}\right) \leq \lambda$.

We will need the following lemma.

LEMMA 3.3. There exists a constant $c=c\left(\theta_{0}, k_{0}\right)>0$ such that for any $p \geq p_{0}, l \geq 2$ and $g_{1}, \ldots, g_{l}$ in $G_{N}$, we have

$$
d\left(\mathbf{o}, g_{1} h^{p} g_{2} \cdots h^{p} g_{l} \mathbf{o}\right) \geq d\left(\mathbf{o}, g_{1} \mathbf{o}\right)+d\left(\mathbf{o}, h^{p} \mathbf{o}\right)+d\left(\mathbf{o}, g_{2} \cdots h^{p} g_{l} \mathbf{o}\right)-c .
$$

Proof. We know that $\mathbf{0} \notin W^{-} \cup W^{+}$, as $G_{N} \mathbf{0} \cap\left(W^{-} \cup W^{+}\right)=\emptyset$ and $\mathrm{Id} \in G_{N}$. By repeatedly applying (ii) and (iv) of Lemma 3.1, we deduce that $h^{p} g_{2} \cdots h^{p} g_{l} \mathbf{0} \in\left(W^{-} \cup W^{+}\right)$, so by (iii) and by Fact 2.1, we deduce the existence of a constant $c_{1}>0$ such that for $l \geq 2$,

$$
\begin{aligned}
d\left(\mathbf{o}, g_{1} h^{p} g_{2} \cdots h^{p} g_{l} \mathbf{o}\right) & =d\left(g_{1}^{-1} \mathbf{o}, h^{p} g_{2} \cdots h^{p} g_{l} \mathbf{o}\right) \\
& \geq d\left(\mathbf{o}, g_{1} \mathbf{o}\right)+d\left(\mathbf{o}, h^{p} g_{2} \cdots h^{p} g_{l} \mathbf{0}\right)-c_{1}
\end{aligned}
$$

and, analogously,

$$
d\left(\mathbf{o}, h^{p} g_{2} \cdots h^{p} g_{l} \mathbf{0}\right) \geq d\left(\mathbf{o}, h^{p} \mathbf{o}\right)+d\left(\mathbf{o}, g_{2} \cdots h^{p} g_{l} \mathbf{o}\right)-c_{1} .
$$

Therefore taking $c=2 c_{1}$ completes the proof.

Proof of Proposition 3.2. Let $p \geq p_{0}$, set $g:=g_{1} h^{p} \cdots h^{p} g_{l}=g_{1}^{\prime} h^{p} \cdots h^{p} g_{l}^{\prime}$ and let $\mathbf{A}:=g_{1} \mathbf{o}, \mathbf{B}:=g_{1} h^{p} \mathbf{o}, \mathbf{A}^{\prime}:=g_{1}^{\prime} \mathbf{o}, \mathbf{B}^{\prime}:=g_{1}^{\prime} h^{p} \mathbf{o}$. By Lemma 3.3, we get

$$
d(\mathbf{o}, g \mathbf{o}) \geq d(\mathbf{o}, \mathbf{A})+d(\mathbf{A}, \mathbf{B})+d(\mathbf{B}, g \mathbf{o})-c,
$$

where the constant $c$ does not depend on $g_{1}, \ldots, g_{l}$ and $l \geq 1$. Applying Fact 2.2, the arc $[\mathbf{o}, \mathbf{A}] \cup[\mathbf{A}, \mathbf{B}] \cup[\mathbf{B}, g \mathbf{o}]$ is included in an $\epsilon$-neighbourhood of $[\mathbf{o}, g \mathbf{o}]$, for some constant $\epsilon$ depending on $c$. Let us now choose $\mathbf{x}, \mathbf{y}, \mathbf{x}^{\prime}, \mathbf{y}^{\prime} \in[\mathbf{o}, g \mathbf{0}]$ such that $d(\mathbf{x}, \mathbf{A}), d(\mathbf{y}, \mathbf{B}), d\left(\mathbf{x}^{\prime}, \mathbf{A}^{\prime}\right)$ and $d\left(\mathbf{y}^{\prime}, \mathbf{B}^{\prime}\right)$ are smaller than $\epsilon$.

Step 1. For $p \gg 0$, we get $\mathbf{x} \in[\mathbf{o}, \mathbf{y}]$ and $\mathbf{x}^{\prime} \in\left[\mathbf{o}, \mathbf{y}^{\prime}\right]$. Actually, we have

$$
\begin{gathered}
|d(\mathbf{o}, \mathbf{x})-d(\mathbf{o}, \mathbf{A})| \leq \epsilon, \\
|d(\mathbf{o}, \mathbf{y})-d(\mathbf{o}, \mathbf{B})| \leq \epsilon, \\
|d(\mathbf{x}, \mathbf{y})-d(\mathbf{A}, \mathbf{B})| \leq 2 \epsilon,
\end{gathered}
$$

with $d(\mathbf{A}, \mathbf{B})=d\left(\mathbf{o}, h^{p} \mathbf{o}\right)=p d(\mathbf{o}, h \mathbf{o})$, and the same inequalities hold, replacing $\mathbf{x}, \mathbf{y}, \mathbf{A}$ and $\mathbf{B}$ with $\mathbf{x}^{\prime}, \mathbf{y}^{\prime}, \mathbf{A}^{\prime}$ and $\mathbf{B}^{\prime}$ respectively. Now, if $\mathbf{y} \in[\mathbf{o}, \mathbf{x}]$, we get $d(\mathbf{o}, \mathbf{x})=d(\mathbf{o}, \mathbf{y})+$ $d(\mathbf{x}, \mathbf{y})$ and so

$$
\begin{aligned}
d(\mathbf{o}, \mathbf{A}) & \geq d(\mathbf{o}, \mathbf{x})-\epsilon \\
& \geq d(\mathbf{o}, \mathbf{y})+d(\mathbf{x}, \mathbf{y})-\epsilon \\
& \geq d(\mathbf{o}, \mathbf{B})+p d(\mathbf{o}, h \cdot \mathbf{o})-4 \epsilon
\end{aligned}
$$


Since $g_{1}$ is minimal and $N$ is normal in $G$, we know that $d(\mathbf{o}, \mathbf{A}) \leq d\left(\mathbf{o}, g_{1} h^{p} \mathbf{o}\right)=d(\mathbf{o}, \mathbf{B})$, and therefore $p \leq 4 \epsilon / d(\mathbf{o}, h \mathbf{o})$. In the following we will fix $p>4 \epsilon / d(\mathbf{0}, h \mathbf{o})$; so, we have $\mathbf{x} \in[\mathbf{o}, \mathbf{y}], \mathbf{x}^{\prime} \in\left[\mathbf{o}, \mathbf{y}^{\prime}\right]$ and, without loss of generality, we assume that $\mathbf{x} \in\left[\mathbf{o}, \mathbf{x}^{\prime}\right]$.

Step 2. When $p \gg 0$, the geodesic segments $\left[\mathbf{x}, \mathbf{x}^{\prime}\right]$ and $\left[\mathbf{y}, \mathbf{y}^{\prime}\right]$ are disjoint. In fact, we have $\overline{\mathbf{A}}=\overline{\mathbf{B}}$, so $\bar{d}(\overline{\mathbf{x}}, \overline{\mathbf{y}}) \leq 2 \epsilon$ and

$$
d(\mathbf{x}, \mathbf{y})-\bar{d}(\overline{\mathbf{x}}, \overline{\mathbf{y}}) \geq d\left(\mathbf{o}, h^{p} \cdot \mathbf{o}\right)-4 \epsilon .
$$

Let $p_{1} \geq 0$ such that $\epsilon^{\prime}:=d\left(\mathbf{o}, h^{p_{1}} \mathbf{o}\right)-4 \epsilon>2 \epsilon$; if $p \geq p_{1}$, we get

$$
d(\mathbf{x}, \mathbf{y}) \geq \bar{d}(\overline{\mathbf{x}}, \overline{\mathbf{y}})+\epsilon^{\prime} .
$$

Now assume, for instance, that $\mathbf{y} \in\left[\mathbf{x}, \mathbf{x}^{\prime}\right]$; we would thus have

$$
\begin{aligned}
d\left(\mathbf{o}, \mathbf{A}^{\prime}\right) & \geq d\left(\mathbf{o}, \mathbf{x}^{\prime}\right)-\epsilon \\
& \geq d(\mathbf{o}, \mathbf{x})+d(\mathbf{x}, \mathbf{y})+d\left(\mathbf{y}, \mathbf{x}^{\prime}\right)-\epsilon \\
& \geq \bar{d}(\overline{\mathbf{o}}, \overline{\mathbf{x}})+\bar{d}(\overline{\mathbf{x}}, \overline{\mathbf{y}})+\bar{d}\left(\overline{\mathbf{y}}, \overline{\mathbf{x}}^{\prime}\right)+\epsilon^{\prime}-\epsilon \\
& \geq \bar{d}\left(\overline{\mathbf{o}}, \overline{\mathbf{x}}^{\prime}\right)+\epsilon^{\prime}-\epsilon \\
& \geq \bar{d}\left(\overline{\mathbf{o}}, \overline{\mathbf{A}}^{\prime}\right)+\epsilon^{\prime}-2 \epsilon
\end{aligned}
$$

and, by the choice of $p_{1}$, we would deduce $\epsilon^{\prime}-2 \epsilon>0$, a contradiction. The same argument proves that $\mathbf{y}^{\prime} \notin\left[\mathbf{x}, \mathbf{x}^{\prime}\right]$.

Finally, to complete the proof of Proposition 3.2, we note that

$$
\begin{aligned}
d\left(\mathbf{A}, \mathbf{A}^{\prime}\right) & \leq d(\mathbf{A}, \mathbf{x})+d\left(\mathbf{x}, \mathbf{x}^{\prime}\right)+d\left(\mathbf{x}^{\prime}, \mathbf{A}^{\prime}\right) \\
& \leq 2 \epsilon+d(\mathbf{x}, \mathbf{y}) \leq 4 \epsilon+d\left(\mathbf{o}, h^{p} \mathbf{o}\right)
\end{aligned}
$$

so it is enough to set $\lambda:=4 \epsilon+d\left(\mathbf{o}, h^{p} \mathbf{o}\right)$.

Proof of Theorem 1.1. Let $h \in N$ be a hyperbolic isometry, and let $\mathbf{0} \in \tilde{h}, G_{N}, p \gg 0, \lambda$ as in Lemma 3.1 and Proposition 3.2. Then consider a maximal $\lambda$-separated net of $G_{N}$, that is, a subset $S$ such that:

- $\quad d\left(a \mathbf{o}, a^{\prime} \mathbf{o}\right)>\lambda$ for all $a, a^{\prime} \in A$;

- $\quad$ for all $g \in G_{N}$ there exists $a \in A: d(g \mathbf{0}, a \mathbf{o}) \leq \lambda$.

Notice that such a subset always exists by the Zorn lemma, since the space of $\lambda$-separated nets forms a partially ordered space where every chain has a majorant. Clearly, we have $\delta_{A}=\delta_{G_{N}}$; that is to say, for any $s \in \mathbb{R}^{+}$, we get

$$
\begin{aligned}
\sum_{g \in G_{N}} \exp (-s d(\mathbf{o}, g \cdot \mathbf{o})) & \leq \sum_{a \in A} \sum_{\substack{g \in G_{N} \\
d(g \mathbf{0}, a \mathbf{0}) \leq \lambda}} \exp (-s d(\mathbf{o}, g \cdot \mathbf{o})) \\
& \leq M \exp (s \lambda) \sum_{a \in A} \exp (-s d(\mathbf{o}, a \mathbf{o}))
\end{aligned}
$$

for $M:=\sharp\left(G \mathbf{o} \cap \mathcal{B}_{\bar{X}}(\mathbf{o}, \lambda)\right)$; so $\delta_{A} \geq \delta_{G_{N}}$, and thus $\delta_{A}=\delta_{G_{N}}$ as $A \subset G_{N}$. Furthermore, the series $\sum_{a \in A} \exp (-s d(\mathbf{0}, a \mathbf{o}))$ diverges at $s=\delta_{A}$ since $\bar{G}$ is divergent. Now, a repeated application of Proposition 3.2 tells us that the evaluation map $\phi: \mathfrak{A}\left(A,\left\{h^{p}\right\}\right) \rightarrow$ $A *\left\{h^{p}\right\} \subset G$ is one-to-one. Then, by Criterion 2.4 we conclude that

$$
\delta_{G} \geq \delta_{A *\left\{h^{p}\right\}}>\delta_{A}=\delta_{G_{N}}=\delta_{\bar{G}} .
$$




\section{Quotients of geometrically finite groups}

We first recall the definition and some well-known properties of geometrically finite groups [4]. Let $G$ be a Kleinian group of $X$ and let $\Lambda_{G}$ its limit set (that is, the set of accumulation points on the boundary at infinity $X(\infty)$ of any orbit $G \mathbf{x}$ of $G$ ). The convex hull $C_{G}$ of $\Lambda_{G}$ is the smallest $G$-invariant closed subset of $X$, and the quotient space $\mathcal{C}_{G}:=C_{G} / G$ is called the Nielsen core of the quotient manifold $\mathcal{X}=G \backslash X$. The group $G$ is geometrically finite if some (or equivalently any) $\epsilon$-neighbourhood of $\mathcal{C}_{G}$ in $\mathcal{X}$ has finite volume.

There are two cases:

- $\quad$ either $\mathcal{C}_{G}$ is compact, and then $G$ is called convex cocompact;

- $\quad$ or $\mathcal{C}_{G}$ is not compact, in which case it can be decomposed into a disjoint union of a compact part $\mathcal{C}_{0}$ and finitely many 'cuspidal ends' $\mathcal{C}_{1}, \ldots, \mathcal{C}_{l}$ : each $\mathcal{C}_{i}$ is isometric to the quotient, by a maximal parabolic group $P_{i} \subset G$, of the intersection between $C_{G}$ and some horoball $\mathcal{H}_{i}$ preserved by $P_{i}$.

Equivalently, $G$ is geometrically finite if its limit set $\Lambda_{G}$ is the union of its radial subset and of finitely many orbits $G \xi_{1}, \ldots, G \xi_{l}$ of points, called bounded parabolic fixed points: each point $\xi_{i}$ is fixed by a maximal parabolic subgroup $P_{i}$ of $G$ (corresponding to an end $\mathcal{C}_{i}$ of $\mathcal{C}_{G}$ ), the group $P_{i}$ preserves the horoballs $\mathcal{H}_{i}$ centred at $\xi_{i}$ with quotient $\mathcal{C}_{i}$, and acts cocompactly on $\partial \mathcal{H}_{i} \cap C_{G}$. Moreover, by Margulis' lemma (see [4]), there exist closed horoballs $\mathcal{H}_{\xi_{1}}, \ldots, \mathcal{H}_{\xi_{l}}$ centred respectively at $\xi_{1}, \ldots, \xi_{l}$, such that all the horoballs $g \mathcal{H}_{\xi_{i}}$, for $1 \leq i \leq l$ and for all $g \in G$, are disjoint or coincide.

We fix a convex Borel fundamental domain $D$ for the action of $G$ in $C_{G}$ and a point $\mathbf{x} \in C_{G}$ such that the geodesic rays $\left[\mathbf{o}, \xi_{1}\right), \ldots,\left[\mathbf{o}, \xi_{l}\right)$ are included in $D$. For each $1 \leq i \leq l$, we set $C_{i}=D \cap \mathcal{H}_{\xi_{i}}$, thus obtaining fundamental domains for the action of the group $P_{i}$ on $\mathcal{H}_{i} \cap C_{G}$; moreover, we may assume that the set $C_{0}=D \backslash\left(\bigcup_{i=1}^{l} C_{i}\right)$ is relatively compact and that $\mathbf{x}$ belongs to its interior. Notice that $\mathcal{C}_{0}$ and the ends $\mathcal{C}_{1}, \ldots, \mathcal{C}_{l}$ of the quotient manifold $\mathcal{C}$ are the projections on $\mathcal{C}_{G}$ of the domains $C_{0}$ and $C_{1}, \ldots, C_{l}$ respectively.

Throughout this section, we will use the following notation. If $\bar{G}=N \backslash G$ is the quotient of a geometrically finite Kleinian group $G$ by the normal subgroup $N$, and $\bar{X}=N \backslash X$, we denote by:

- $\quad \bar{C}_{G}, \bar{D}, \bar{C}_{0}, \bar{C}_{1}, \ldots, \bar{C}_{l}$ the projections of $C_{G}, D, C_{0}, C_{1}, \ldots, C_{l}$ on $\bar{X}$ respectively (notice that, as the projection $\pi: X \rightarrow \bar{X}$ is equivariant with respect to $\rho$ : $G \rightarrow \bar{G}$, we have that $\bar{G} \bar{D}=\bar{C}_{G}$ );

- $\quad \mathcal{B}_{\bar{G}}(\overline{\mathbf{x}}, R)$ and $\mathcal{A}_{\bar{G}}(\overline{\mathbf{x}}, \alpha, R)$ respectively the ball of radius $R$ and the 'annulus' of the same radius and width $2 \Delta$ in the orbit $\bar{G}$,

$$
\begin{gathered}
\mathcal{B}_{\bar{G}}(\overline{\mathbf{x}}, R):=\{\overline{\mathbf{y}} \in \bar{G} \cdot \overline{\mathbf{x}} \mid \bar{d}(\overline{\mathbf{x}}, \overline{\mathbf{y}}) \leq R\}, \\
\mathcal{A}_{\bar{G}}(\overline{\mathbf{x}}, \Delta, R):=\{\overline{\mathbf{y}} \in \bar{G} \cdot \overline{\mathbf{x}} \mid R-\Delta<\bar{d}(\overline{\mathbf{x}}, \overline{\mathbf{y}}) \leq R+\Delta\}
\end{gathered}
$$

- $\quad v_{\bar{G}}(\overline{\mathbf{x}}, R)=\sharp \mathcal{B}_{\bar{G}}(\overline{\mathbf{x}}, R)$ and $v_{\bar{G}}(\overline{\mathbf{x}}, \Delta, R)=\sharp \mathcal{A}_{\bar{G}}(\overline{\mathbf{x}}, \Delta, R)$ the growth functions for balls and annuli;

- $\quad w_{\bar{G}}^{s}(\overline{\mathbf{x}}, \Delta, R)=e^{-s R} v_{\bar{G}}(\overline{\mathbf{x}}, \Delta, R)$ the growth function renormalized by $e^{s R}$. 
Notice that, as

$$
\frac{1}{2 \Delta} \sum_{n \geq 0} \sum_{g \in \mathcal{A}_{\bar{G}}(\overline{\mathbf{x}}, \Delta, n)} e^{-s(n+\Delta)} \leq \mathcal{P}_{\bar{G}}(\overline{\mathbf{x}}, s) \leq \sum_{n \geq 0} \sum_{g \in \mathcal{A}_{\bar{G}}(\overline{\mathbf{x}}, \Delta, n)} e^{-s(n-\Delta)},
$$

the series $\mathcal{P}_{\bar{G}}(\overline{\mathbf{x}}, s)$ and $\hat{\mathcal{P}}_{\bar{G}}(\overline{\mathbf{x}}, s)=\sum_{n \geq 0} e^{-s n} v_{\bar{G}}(\overline{\mathbf{x}}, \Delta, n)$ have the same critical exponent $\delta_{\bar{G}}$ and the same behaviour for $s=\delta_{\bar{G}}$. Moreover, from this it easily follows that we also have

$$
\delta_{\bar{G}}=\limsup _{R \rightarrow+\infty} \frac{1}{R} \ln v_{\bar{G}}(\overline{\mathbf{x}}, \Delta, R) .
$$

We will prove the following (quasi-)submultiplicative result, which is the key to the divergence criterion. As far as the authors are aware, the submultiplicativity of the function $v_{\bar{G}}(\overline{\mathbf{x}}, \Delta, R)$ is due, in the cocompact case, to Robert [14] (unpublished).

Proposition 4.1. Let $\bar{G}=N \backslash G$ be the quotient of a geometrically finite Kleinian group $G$, and let $\overline{\mathbf{x}} \in \bar{X}=N \backslash X$. There exists $\Delta>0$ such that:

- if $G$ is convex cocompact, then

$$
v_{\bar{G}}(\overline{\mathbf{x}}, \Delta, A+B) \leq v_{\bar{G}}(\overline{\mathbf{x}}, \Delta, A) \cdot v_{\bar{G}}(\overline{\mathbf{x}}, \Delta, B) \quad \text { for all } A, B \gg 0
$$

- $\quad$ if $G$ satisfies $(P G C)$, i.e. $\delta_{\bar{P}}<\delta_{\bar{G}}$ for every parabolic subgroup $P<G$, then

$$
\begin{gathered}
w_{\bar{G}}^{s}(\overline{\mathbf{x}}, \Delta, A+B) \leq c \cdot\left(\sum_{a=1}^{\lceil A\rceil} w_{\bar{G}}^{s}(\overline{\mathbf{x}}, \Delta, a)\right) \cdot\left(\sum_{b=1}^{\lceil B\rceil} w_{\bar{G}}^{s}(\overline{\mathbf{x}}, \Delta, b)\right) \\
\text { for all } A, B \gg 0
\end{gathered}
$$

for any fixed $s>\max \left\{\delta_{\bar{P}_{1}}, \ldots, \delta_{\bar{P}_{l}}\right\}$ and for some constant $c=c(\bar{G}, s)$.

Proof of Proposition 4.1. Fix $\Delta_{0} \geq 2$ greater than the diameter of the compact region $\bar{C}_{0}$ of the fundamental domain $\bar{D}$ for the action of $\bar{G}$ on $\bar{C}$. We shall show that

$$
\mathcal{A}_{\bar{G}}\left(\overline{\mathbf{x}}, 3 \Delta_{0}, A+B\right) \subset \bigcup_{\bar{g} \overline{\mathbf{x}} \in \mathcal{A}_{\bar{G}}\left(\overline{\mathbf{x}}, 3 \Delta_{0}, A\right)} \mathcal{A}_{\bar{G}}\left(\bar{g} \overline{\mathbf{x}}, 3 \Delta_{0}, B\right)
$$

in the convex cocompact case, while

$$
\mathcal{A}_{\bar{G}}\left(\overline{\mathbf{x}}, 3 \Delta_{0}, A+B\right) \subset \bigcup_{\substack{a, b>1 \\ a \leq\lceil A\rceil, b \leq\lceil B\rceil}} \bigcup_{(g, p) \in \mathcal{I}_{a, b}} \mathcal{A}_{\bar{G}}\left(\bar{g} \bar{p} \overline{\mathbf{x}}, 3 \Delta_{0}, b\right)
$$

in the geometrically finite case, where

$$
\begin{gathered}
\mathcal{I}_{a, b}=\left\{(\bar{g}, \bar{p}) \mid \bar{g} \overline{\mathbf{x}} \in \mathcal{A}_{\bar{G}}\left(\overline{\mathbf{x}}, 3 \Delta_{0}, a\right), \bar{p} \overline{\mathbf{x}} \in \mathcal{B}_{\bar{P}_{i}}\left(\overline{\mathbf{x}}, A+B-a-b+3 \Delta_{0}\right),\right. \\
i=1, \ldots, n\} .
\end{gathered}
$$

Actually, let $\overline{\mathbf{y}} \in \mathcal{A}_{\bar{G}}\left(\overline{\mathbf{x}}, 3 \Delta_{0}, A+B\right)$ : we write $\bar{d}(\overline{\mathbf{x}}, \overline{\mathbf{y}})=A+B+3 \lambda$ with $-\Delta_{0}<$ $\lambda \leq \Delta_{0}$, and we consider a minimizing geodesic $[\overline{\mathbf{x}}, \overline{\mathbf{y}}]$ and a point $\overline{\mathbf{z}} \in[\overline{\mathbf{x}}, \overline{\mathbf{y}}]$ such that

$$
\bar{d}(\overline{\mathbf{x}}, \overline{\mathbf{z}})=A+\frac{3}{2} \lambda, \quad \bar{d}(\overline{\mathbf{z}}, \overline{\mathbf{y}})=B+\frac{3}{2} \lambda .
$$

We lift $\overline{\mathbf{x}}, \overline{\mathbf{y}}, \overline{\mathbf{z}}$ and $[\overline{\mathbf{x}}, \overline{\mathbf{y}}]$ to points $\mathbf{x}, \mathbf{y}, \mathbf{z} \in[\mathbf{x}, \mathbf{y}]$ in $X$, still satisfying $d(\mathbf{x}, \mathbf{z})=A+\frac{3}{2} \lambda$ and $d(\mathbf{z}, \mathbf{y})=B+\frac{3}{2} \lambda$. As $C_{G}$ is convex, we know that $[\mathbf{x}, \mathbf{y}]$ and $\mathbf{z}$ lie in $C_{G}$, hence $[\overline{\mathbf{x}}, \overline{\mathbf{y}}]$ and $\overline{\mathbf{z}}$ lie in $\bar{C}_{G}$. 
Now if $G$ is convex cocompact we have $\bar{D}=\bar{C}_{0}$, so $\overline{\mathbf{z}} \in \bar{G} \bar{C}_{0}$, hence we can find some $\bar{g} \in \bar{G}$ such that $\bar{d}(\overline{\mathbf{z}}, \bar{g} \overline{\mathbf{x}}) \leq \Delta_{0}$. Therefore $\bar{g} \overline{\mathbf{x}} \in \mathcal{A}_{\bar{G}}\left(\overline{\mathbf{x}}, 3 \Delta_{0}, A\right)$ and $\overline{\mathbf{y}} \in \mathcal{A}_{\bar{G}}\left(\bar{g} \overline{\mathbf{x}}, 3 \Delta_{0}, B\right)$, so (4) holds.

On the other hand, if $\bar{G}$ is geometrically finite, $\overline{\mathbf{z}}$ may belong to $\bar{G} \bar{C}_{0}$ or to $\bar{C}_{G} \backslash \bar{G} \bar{C}_{0}=$ $\bigcup_{i=1}^{n} \bar{G} \bar{C}_{i}$. In the first case, the argument above shows that $\overline{\mathbf{z}}$ belongs to the right-hand side of (5) (as (4) implies (5)). Otherwise, the lift $\mathbf{z}$ of $\overline{\mathbf{z}}$ belongs to some horoball $\mathcal{H}$ fixed by the conjugate of one maximal parabolic subgroup $P_{i}$. Thus, we can cut the geodesic $[\mathbf{x}, \mathbf{y}]$ into three pieces $[\mathbf{x}, \mathbf{u}] \cup[\mathbf{u}, \mathbf{v}] \cup[\mathbf{v}, \mathbf{y}]$, where $\mathbf{z} \in[\mathbf{u}, \mathbf{v}]=[\mathbf{x}, \mathbf{y}] \cap \mathcal{H}$ and $\mathbf{u} \in g C_{0}, \mathbf{v} \in g p C_{0}$, for some $g \in G$ and $p \in P_{i}$. We then consider the projections $\bar{g}, \bar{p}$ in $\bar{G}$ and $\overline{\mathbf{u}} \in \bar{g} \bar{C}_{0}, \overline{\mathbf{v}} \in \bar{p} \bar{C}_{0}$ (so $\left.\bar{d}(\bar{g} \overline{\mathbf{x}}, \overline{\mathbf{u}}) \leq \Delta_{0}, \bar{d}(\bar{g} \bar{p} \overline{\mathbf{x}}, \overline{\mathbf{v}}) \leq \Delta_{0}\right)$. Setting $\alpha=\bar{d}(\overline{\mathbf{x}}, \overline{\mathbf{u}})-$ $\frac{3}{2} \lambda, \beta=\bar{d}(\overline{\mathbf{y}}, \overline{\mathbf{v}})-\frac{3}{2} \lambda$ and $a=\lceil\alpha\rceil \leq\lceil A\rceil, b=\lceil\beta\rceil \leq\lceil B\rceil$, we have:

(i) $\bar{g} \overline{\mathbf{x}} \in \mathcal{A}_{\bar{G}}\left(\overline{\mathbf{x}}, 3 \Delta_{0}, a\right)$;

(ii) $\overline{\mathbf{y}} \in \mathcal{A}_{\bar{G}}\left(\overline{g p} \overline{\mathbf{x}}, 3 \Delta_{0}, b\right)$;

(iii) $\bar{d}(\overline{\mathbf{x}}, \bar{p} \overline{\mathbf{x}})=\bar{d}(\bar{g} \overline{\mathbf{x}}, \overline{g p} \overline{\mathbf{x}}) \leq \bar{d}(\overline{\mathbf{u}}, \overline{\mathbf{v}})+2 \Delta_{0} \leq(A-a)+(B-b)+3 \Delta_{0}$, which proves (5).

In the convex cocompact case, from (4) we immediately infer (2).

In the general geometrically finite case, from (5) we deduce

$$
\begin{aligned}
v_{\bar{G}}\left(\overline{\mathbf{x}}, 3 \Delta_{0}, A+B\right) \leq & \sum_{a=0}^{\lceil A\rceil} \sum_{b=0}^{\lceil B\rceil} \sum_{i=1}^{l} v_{\bar{G}}\left(\overline{\mathbf{x}}, 3 \Delta_{0}, a\right) v_{\bar{G}}\left(\overline{\mathbf{x}}, 3 \Delta_{0}, b\right) \\
& \times v_{\bar{P}_{i}}\left(\overline{\mathbf{x}}, A+B-a-b+3 \Delta_{0}\right) .
\end{aligned}
$$

Since we know that $v_{\bar{P}_{i}}(\overline{\mathbf{x}}, R) \leq c_{0} e^{s R}$ for any fixed $s>\max \left\{\delta_{P_{1}}, \ldots, \delta_{P_{l}}\right\}$ (for some constant $\left.c_{0}=c_{0}(\bar{G}, s)\right)$, we obtain

$$
\begin{aligned}
v_{\bar{G}}(\overline{\mathbf{x}}, \Delta, A+B) e^{-s(A+B)} & \leq c \cdot \sum_{a=0}^{\lceil A\rceil} \sum_{b=0}^{\lceil B\rceil} e^{-s A} v_{\bar{G}}(\overline{\mathbf{x}}, \Delta, a) e^{-s B} v_{\bar{G}}(\overline{\mathbf{x}}, \Delta, b) e^{s(A+B-a-b)} \\
& \leq c \cdot\left(\sum_{a=0}^{\lceil A\rceil} e^{-s a} v_{\bar{G}}(\overline{\mathbf{x}}, \Delta, a)\right) \cdot\left(\sum_{b=0}^{\lceil B\rceil} e^{-s b} v_{\bar{G}}(\overline{\mathbf{x}}, \Delta, b)\right)
\end{aligned}
$$

that is, formula (3).

We are now ready to prove Theorems 1.2 and 1.4.

Proof of Theorem 1.2. Beginning with the convex cocompact case, we apply the following elementary lemma to the sequence $v_{n}:=v_{\bar{G}}(\overline{\mathbf{x}}, \Delta, n)$, which satisfies the submultiplicative condition (2) of Proposition 4.1 above.

LEMMA 4.2. (Fekete [13]) Let $\left(v_{n}\right)_{n \geq 1}$ be a sequence of positive numbers such that

$$
v_{n+m} \leq v_{n} v_{m} \text { for all } n, m \geq n_{0} .
$$

Then $\lim _{n \rightarrow \infty}(1 / n) \ln v_{n}=L \in \mathbb{R} \cup\{-\infty\}$ and $v_{n} \geq e^{L n}$ for all $n \geq n_{0}$.

As $\lim _{R \rightarrow \infty}(1 / R) \ln v_{\bar{G}}(\overline{\mathbf{x}}, \Delta, R)=\delta_{\bar{G}}$, this shows that $v_{\bar{G}}(\overline{\mathbf{x}}, \Delta, n) \geq e^{\delta_{\bar{G}} n}$ for $n \gg 0$, so that the series $\widehat{\mathcal{P}}_{\bar{G}}(\overline{\mathbf{x}}, s)$ (and therefore $\mathcal{P}_{\bar{G}}(\overline{\mathbf{x}}, s)$ ) is divergent.

Turning to the geometrically finite case, if the group $G$ contains parabolic elements, we again use Proposition 4.1, with a refined version of Fekete's lemma. 
LEMMA 4.3. Let $\left(w_{n}\right)_{n \geq 1}$ be a sequence of positive numbers such that

$$
w_{n+m} \leq c \cdot\left(\sum_{k=1}^{n} w_{k}\right) \cdot\left(\sum_{k=1}^{m} w_{k}\right) \text { for all } n, m \geq n_{0}
$$

for some positive constant $c$. Then, setting $W_{n}=\sum_{k=1}^{n} w_{k}$, we have:

(i) $\lim _{n \rightarrow \infty}(1 / n) \ln W_{n}=L \in \mathbb{R} \cup\{-\infty\}$ and $W_{n} \geq e^{L n} / 2$ cn for all $n \gg 0$;

(ii) if $L>0$ then the series $\sum_{n} W_{n} e^{-s n}$ and $\sum_{n} w_{n} e^{-s n}$ have the same critical exponent $L$, and for $s=L$ they are divergent.

Let us for a moment assume Lemma 4.3 and conclude the proof of Theorem 1.2. As $\bar{G}$ satisfies the parabolic gap condition by assumption, we can choose $s$ such that $\delta_{\bar{P}}<$ $s<\delta_{\bar{G}}$ for every parabolic subgroup $P \subset G$. Then, by Proposition 4.1 , the sequence $w_{n}:=w_{\bar{G}}^{s}(\overline{\mathbf{x}}, \Delta, n)=e^{-s n} v_{\bar{G}}(\overline{\mathbf{x}}, \Delta, n)$ satisfies the assumption of the previous lemma, with $L=\lim _{n \rightarrow \infty}(1 / n) \ln w_{\bar{G}}^{s}(\overline{\mathbf{x}}, \Delta, n)=\delta_{\bar{G}}-s>0$ by (1). Hence, the series

$$
\sum_{n} w_{n} e^{-L n}=\sum_{n} e^{-s n} v_{\bar{G}}(\overline{\mathbf{x}}, \Delta, n) e^{-\left(\delta_{\bar{G}}-s\right) n}=\widehat{\mathcal{P}}_{\bar{G}}\left(\overline{\mathbf{x}}, \delta_{\bar{G}}\right)
$$

diverges, as well as $\mathcal{P}_{\bar{G}}\left(\overline{\mathbf{x}}, \delta_{\bar{G}}\right)$.

Proof of Lemma 4.3. We prove the lemma when $n_{0}=1$ (the argument for $n_{0}>1$ being analogous), and we may assume that $c>1$. We set

$$
\tilde{W}_{n}:=1+W_{1}+\cdots+W_{n}
$$

and

$$
v_{n}:=c w_{n}, \quad V_{n}:=\sum_{k=1}^{n} v_{k}=c W_{n}, \quad \tilde{V}_{n}:=c \tilde{W}_{n} \geq 1+\sum_{k=1}^{n} V_{k},
$$

so we obtain

$$
v_{n+m} \leq V_{n} V_{m} \text { for all } n, m \geq 1 .
$$

This in turn yields $V_{n+m} \leq V_{n} \tilde{V}_{m}$ and, consequently,

$$
\tilde{V}_{n+m}=\tilde{V}_{n}+V_{n+1}+\cdots+V_{n+m} \leq \tilde{V}_{n} \tilde{V}_{m} \text { for all } n, m \geq 1 .
$$

So $\left(\tilde{V}_{n}\right)_{n \geq 1}$ satisfies the assumption of Fekete's lemma. Hence the sequences $\left(\left(\ln \tilde{V}_{n}\right) / n\right)_{n}$ and $\left(\left(\ln \tilde{W}_{n}\right) / n\right)_{n}$ converge to some $L \in \mathbb{R} \cup\{-\infty\}$ with $\tilde{V}_{n} \geq e^{L n}$ and $\tilde{W}_{n} \geq(1 / c) e^{L n}$ for $n$ large enough. As $(1 / n)\left(\tilde{W}_{n}-1\right) \leq W_{n} \leq \tilde{W}_{n}$, the sequence $\left(\left(\ln W_{n}\right) / n\right)_{n}$ converges also to $L$ and the series $\sum_{n} e^{-s n} W_{n}$ has critical exponent equal to $L$. Moreover, for $n$ large enough, we get

$$
W_{n} \geq 1+\frac{1}{n}\left(\tilde{W}_{n}-1\right) \geq \frac{1}{2 c n} e^{L n}
$$

so the series $\sum_{n} e^{-s n} W_{n}$ diverges at $s=L$. Now

$$
\sum_{n \geq 1} e^{-s n} W_{n}=\sum_{k \geq 1}\left(\sum_{n=k}^{\infty} e^{-s n}\right) w_{k}=\sum_{k \geq 1}\left(\frac{e^{-s k}}{1-e^{-s}}\right) w_{k}=\left(\frac{1}{1-e^{-s}}\right) \sum_{k \geq 1} e^{-s k} w_{k},
$$

so the series $\sum_{k} e^{-s k} w_{k}$ has the same critical exponent $L$ as $\sum_{n} e^{-s n} W_{n}$ and, if $L>0$, it diverges at $s=L$. 
Proof of Theorem 1.4. Let $G$ be a geometrically finite group of $X$ satisfying the parabolic gap condition, and let $\bar{G}$ be a proper quotient of $G$. There are two cases to consider:

- $\quad$ either there exists a parabolic subgroup $P<G$ such that $\delta_{\bar{P}}=\delta_{\bar{G}}$, and then $\delta_{\bar{G}} \leq$ $\delta_{P}<\delta_{G}$ by assumption;

- $\quad$ or $\delta_{\bar{P}}<\delta_{\bar{G}}$ for all parabolic subgroups $P<G$ (or $G$ is convex cocompact), in which case Theorem 1.2 ensures that $\bar{G}$ is divergent, and we deduce from Theorem 1.1 that $\delta_{\bar{G}}<\delta_{G}$.

\section{Examples}

We will consider here geometrically finite groups $G$ which are free products of Kleinian groups $A, B$ in Schottky position. Recall that two Kleinian groups $A$ and $B$ are in Schottky position if there exist two disjoint open subsets $U_{A}$ and $U_{B}$ in $X(\infty)$ such that

$$
a\left(X(\infty) \backslash U_{A}\right) \subset U_{A} \quad \text { and } \quad b\left(X(\infty) \backslash U_{B}\right) \subset U_{B} \text { for all } a \in A^{*}, b \in B^{*},
$$

and for some $\mathbf{x} \in X$ the angle $\theta:=\inf _{\xi \in U_{A}, \eta \in U_{B}} L_{\mathbf{x}}(\xi, \eta)>0$. We will say that $A$ and $B$ are in $(\mathbf{x}, \theta)$-Schottky position when the point $x$ and the value of $\theta$ are of importance.

By Klein's table-tennis lemma, two Kleinian groups $A, B$ in Schottky position generate a discrete subgroup of $\operatorname{Isom}(X)$ which is the free product of $A$ and $B$ (see, for instance, $[8]$ ). Any $g \in A * B$ can then be uniquely written as the product $g=g_{1} \cdots g_{n}$, where $\left(g_{i}\right)_{n \geq 1}$ is an admissible sequence with respect to $(A, B)$ (see [3] for more details about groups of this kind). We shall denote by $\rho_{A}, \rho_{B}$ the natural projections of $G=A * B$ on its factors.

Moreover, notice that, when $A, B$ are in $(\mathbf{x}, \theta)$-Schottky position, we can find a positive constant $c_{k_{0}}(\theta)$ (depending on $\theta$ and on the upper bound $k_{0}$ of the curvature of $X$ ) such that for every $\left(g_{1}, \ldots, g_{n}\right) \in \mathfrak{A}(A, B)$ we have

$$
d\left(\mathbf{x}, g_{1} \cdots g_{n} \mathbf{x}\right) \geq \sum_{i=1}^{n} d\left(\mathbf{x}, g_{i} \mathbf{x}\right)-(n-1) c_{k_{0}}(\theta) .
$$

We shall then say that $B$ is $c_{k_{0}}(\theta)$-sparse if

$$
d(\mathbf{x}, b \mathbf{x}) \geq 2 c_{k_{0}}(\theta) \quad \text { for all } b \in B^{*} ;
$$

this condition implies that, for any $\left(g_{1}, \ldots, g_{n}\right) \in \mathfrak{A}(A, B)$, we have

$$
d\left(\mathbf{x}, g_{1} \cdots g_{n} \mathbf{x}\right) \geq \sum_{g_{i} \in A} d\left(\mathbf{x}, g_{i} \mathbf{x}\right)
$$

From this, we deduce the following preliminary lemma.

Lemma 5.1. Let $G=A * B$ be the free product of two Kleinian groups of $X$ in $(\mathbf{x}, \theta)$ Schottky position, and assume that $B$ is $c_{k_{0}}(\theta)$-sparse. Let $N$ be a normal subgroup of $G$. Then

$$
\bar{d}(\overline{\mathbf{x}}, \bar{g} \overline{\mathbf{x}}) \geq \inf _{g^{\prime} \in \rho_{A}(N g)} d\left(\mathbf{x}, g^{\prime} \mathbf{x}\right) .
$$

As a consequence, if $\rho_{A}(N)=\{\mathrm{Id}\}$, then $\delta_{A}=\delta_{\bar{A}}$. 
Proof. The class $\bar{g}=N g$ satisfies

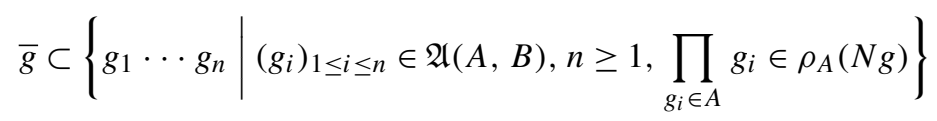

so, by (6) and by the triangular inequality,

$$
\begin{aligned}
\bar{d}(\overline{\mathbf{x}}, \bar{g} \overline{\mathbf{x}}) & \geq \inf \left\{\sum_{g_{i} \in A} d\left(\mathbf{x}, g_{i} \mathbf{x}\right) \mid\left(g_{i}\right)_{1 \leq i \leq n} \in \mathfrak{A}(A, B), n \geq 1, \prod_{g_{i} \in A} g_{i} \in \rho_{A}(N g)\right\} \\
& \geq \inf \left\{d\left(\mathbf{x}, g^{\prime} \mathbf{x}\right) \mid\left(g_{i}\right)_{1 \leq i \leq n} \in \mathfrak{A}(A, B), n \geq 1, g^{\prime}=\prod_{g_{i} \in A} g_{i} \in \rho_{A}(N g)\right\}
\end{aligned}
$$

which proves (i). On the other hand, from (i) we deduce that if $\rho_{A}(N)=\{\operatorname{Id}\}$ then

$$
\bar{d}(\overline{\mathbf{x}}, \bar{a} \overline{\mathbf{x}}) \geq d\left(\mathbf{x}, \rho_{A}(a) \cdot \mathbf{x}\right)=d(\mathbf{x}, a \mathbf{x})
$$

for every $a \in A$, hence $\bar{d}(\overline{\mathbf{x}}, \bar{a} \overline{\mathbf{x}})=d(\mathbf{x}, a \mathbf{x})$; thus the quotient map $\rho_{A}$ gives an isometry between the orbits $A \mathbf{x}$ and $\bar{A} \overline{\mathbf{x}}$, which implies that $\delta_{A}=\delta_{\bar{A}}$.

5.1. A convergent quotient $\bar{G}$ with $\delta_{\bar{G}}=\delta_{G}$. We take a Kleinian group $G$ of $\mathbb{H}^{2}$ generated by two parabolic isometries $a$ and $b$ acting on $\mathbb{H}^{2}$ with fixed points $\xi_{a} \neq \xi_{b}$ in $\mathbb{H}^{2}(\infty)$, with $A=\langle a\rangle$ and $B=\langle b\rangle$ in $(\mathbf{x}, \theta)$-Schottky position. By suitably modifying the metric of $G \backslash \mathbb{H}^{2}$ along the cusp associated with $A$, we can make $G$ become a convergent, geometrically finite Kleinian group of a new, variable curvature CartanHadamard manifold $X$ with pinched negative curvature. The details of this construction can be found in [6]: first, the authors construct a 'convergent cusp', associated with the subgroup $A$; then, they show that taking $B$ with $\delta_{B}<\delta_{A}$ and replacing the generators $a, b$ with $a^{m}$ and $b^{m}$ for $m$ large enough, they can make $G$ convergent with critical exponent $\delta_{G}=\delta_{A}$. The group $G$ will still be a $\theta$-Schottky product of the two maximal parabolic subgroups $A, B$ and, up to replacing $b$ with $b^{m}$, for $m \gg 0$ we may as well assume that $B$ is $c_{k_{0}}(\theta)$-sparse.

Now, if we take $N$ equal to the normal closure of $B$, we have $\bar{G}=N \backslash G \cong \bar{A}=A$ and $\rho_{A}(N)=\{\mathrm{Id}\}$; so, by Lemma 5.1, we deduce that $\delta_{\bar{G}}=\delta_{\bar{A}}=\delta_{A}=\delta_{G}$. Also notice that $\bar{G}$ is convergent, by Theorem 1.1 .

5.2. A convergent quotient $\bar{G}$, with $(P G C)$ holding for $G$ but not for $\bar{G}$. This is the simplest case, and occurs for instance when taking the quotient by $N$ collapses $G$ and a parabolic subgroup to the same group. We take $G=A * B$ for two parabolic subgroups $A$ and $B$ as above ( $A$ convergent and $B c_{k_{0}}(\theta)$-sparse), but here we let both $\delta_{A}, \delta_{B}<\delta_{G}$. So, in this case, $G$ satisfies $(P G C)$ and is divergent (by Theorem 1.2, or by [6]). Again we define $N=\left\langle\langle B\rangle\right.$ so that $\bar{G}=\bar{A}$ and $\delta_{\bar{G}}=\delta_{\bar{A}}$ by Lemma 5.1 ; therefore $(P G C)$ does not hold for $\bar{G}$. Notice that $\bar{G}$ is convergent as its orbit on $\bar{X}$ is isometric to the orbit of the convergent group $A$ on $X$.

5.3. A divergent quotient $\bar{G}$, with (PGC) holding for $\bar{G}$ but not for $G$. In [6], the authors show how to produce, in dimension greater than 2 , two Kleinian groups $A, B$ 
of a Cartan-Hadamard manifold $X$ with pinched negative curvature $k(X) \leq-k_{0}^{2}$ in $(x, \theta)$ Schottky position, such that:

(i) $B$ is parabolic abelian of rank two, generated by $p, p^{\prime}$;

(ii) $G=A * B$ is geometrically finite, with maximal parabolic subgroups conjugated to $B$;

(iii) the groups $B$ and $G$ are convergent, with $\delta_{B}=\delta_{G}$.

It is also straightforward to check that for $B$ we have

$$
d\left(\mathbf{x}, p^{k} p^{\prime} \mathbf{x}\right) \geq d\left(\mathbf{x}, p^{k} \mathbf{x}\right) \quad \text { for all } k, l \in \mathbb{Z} .
$$

Without essentially modifying this construction we can also make

$$
\delta_{\langle p\rangle}<\delta_{A} .
$$

To obtain this condition, we may for instance choose two hyperbolic isometries $h, h^{\prime}$ with fixed points distinct from each other and different from $\xi_{p}=\xi_{p^{\prime}}$, and then set $A=\left\langle h^{n} p h^{-n}, h^{\prime n}\right\rangle$; then $A$ and $B$ will be in $(\mathbf{x}, \theta)$-Schottky position for $n \gg 0$, and (8) will be satisfied by Criterion 2.4 .

Up to taking sufficiently high powers of $p, p^{\prime}, h, h^{\prime}$ we may furthermore assume that $A$ and $B$ are $c_{k_{0}}(\theta)$-sparse.

Now consider the normal subgroup $N$ generated by $p^{\prime}$ : we have $\bar{G} \cong A *\langle p\rangle, \bar{A} \cong A$, $\bar{B} \cong\langle p\rangle$. Since $\rho_{B}(N)=\left\langle p^{\prime}\right\rangle$ and $A$ is $\theta$-sparse, by Lemma 5.1 and by (7) we get

$$
\bar{d}\left(\overline{\mathbf{x}}, \bar{p}^{k} \overline{\mathbf{x}}\right) \geq \inf _{g \in\left\langle p^{\prime}\right\rangle p^{k}} d(\mathbf{x}, g \mathbf{x})=\inf _{l \in \mathbb{Z}} d\left(\mathbf{x}, p^{k} p^{\prime l} \mathbf{x}\right)=d\left(\mathbf{x}, p^{k} \mathbf{x}\right) .
$$

So $\bar{d}\left(\overline{\mathbf{x}}, \bar{p}^{k} \overline{\mathbf{x}}\right)=d\left(\mathbf{x}, p^{k} \mathbf{x}\right)$ for all $k$, and $\delta_{\bar{B}}=\delta_{\langle p\rangle}$. Moreover, since $\rho_{A}(N)=\{\operatorname{Id}\}$ and $B$ is $\theta$-sparse, Lemma 5.1 also yields $\delta_{A}=\delta_{\bar{A}}$. It follows that $\bar{G}$ satisfies $(P G C)$ as

$$
\delta_{\bar{B}}=\delta_{\langle p\rangle}<\delta_{A}=\delta_{\bar{A}} \leq \delta_{\bar{G}} .
$$

Acknowledgements. The authors thank the referee for further valuable suggestions.

\section{REFERENCES}

[1] G. N. Arzhantseva and I. G. Lysenok. Growth tightness for word hyperbolic groups. Math. Z. 241(3) (2002), 597-611.

[2] I. K. Babenko. Asymptotic invariants of smooth manifolds. Russian Acad. Sci. Izv. Math. 41(1) (1993), 1-38.

[3] M. Babillot and M. Peigné. Asymptotic laws for geodesic homology on hyperbolic manifolds with cusps. Bull. Soc. Math. France 134(1) (2006), 119-163.

[4] B. Bowditch. Geometrical finiteness with variable negative curvature. Duke Math. J. 77 (1995), $229-274$.

[5] K. Corlette and A. Iozzi. Limit sets of isometry groups of exotic hyperbolic spaces. Trans. Amer. Math. Soc. 351(4) (1999), 1507-1530.

[6] F. Dal'bo, J. P. Otal and M. Peigné. Séries de Poincaré des groupes géométriquement finis. Israel J. Math. 118 (2000), 109-124.

[7] F. Dal'bo, M. Peigné, J. C. Picaud and A. Sambusetti. On the growth of non-uniform lattices in pinched negatively curved manifolds. J. Reine Angew. Math. 627 (2009), 31-52. 
[8] P. de la Harpe. Topics in Geometric Group Theory (Chicago Lectures in Mathematics). Chicago University Press, Chicago, 2000.

[9] E. Ghys and P. de la Harpe. Sur les groupes hyperboliques d'après Mikhael Gromov. Birkhäuser, Basel, 1990.

[10] R. Grigorchuk and P. de la Harpe. On problems related to growth, entropy and spectrum in group theory. J. Dyn. Control Syst. 3(1) (1997), 51-89.

[11] P. J. Nicholls. The Ergodic Theory of Discrete Groups (London Mathematical Society Lecture Note Series, 143). Cambridge University Press, Cambridge, 1989.

[12] J. P. Otal and M. Peigné. Principe variationnel et groupes Kleiniens. Duke Math. J. 125(1) (2004), 15-44.

[13] G. Pólya and G. Szegö. Problems and Theorems in Analysis, Vol. I \& II (Grundlehren der mathematischen Wissenschaften, 193 and 216). Springer, Berlin, 1972 \& 1976.

[14] G. Robert. Comptage pour des groupes co-compacts d'isométries d'un espace hyperbolique au sens de Gromov. Preprint.

[15] T. Roblin. Ergodicité et equidistribution en courbure négative (Mémoires de la Société Mathématique de France (N.S.), 95). Société Mathématique de France, Paris, 2003.

[16] A. Sambusetti. Growth tightness of surfaces groups. Expositiones Mathematicae 20 (2002), 335-363.

[17] A. Sambusetti. Growth tightness of free and amalgamated products. Ann. Sci. École Norm. Sup. (4) série 35 (2002), 477-488.

[18] A. Sambusetti. Asymptotic properties of coverings in negative curvature. Geom. Topol. 12(1) (2008), 617-637.

[19] D. Sullivan. The density at infinity of a discrete group of hyperbolic motions. Inst. Hautes Études Sci. Publ. Math. 50 (1979), 171-202.

\section{СамвRIDGE JOURNALS}

